\title{
TÉCNICAS ROTATORIA Y CONVENCIONAL PARA PULPECTOMIAS Y SU EFECTO EN EL TIEMPO OPERATORIO Y ANSIEDAD EN PREESCOLARES.
}

\section{ROTATIONAL AND CONVENTIONAL TECHNIQUES FOR PULPECTOMIES AND THEIR EFFECT ON OPERATING TIME AND ANXIETY IN PRESCHOOLS.}

\author{
Mamani-Cori Vilma. ${ }^{* 1}$ Padilla-Cáceres Tania. ${ }^{2}$ Barreda-Salinas Claudia. $^{3}$ \\ ${ }^{1}$ Doctora en Ciencias de la Salud, Especialista en Odontopediatría-Docente Universidad Nacional del Altiplano, Puno-Perú. \\ 2 Doctora en Ciencias de la Salud, Especialista en Odontopediatría.-Docente Universidad Nacional del Altiplano, Puno-Perú. \\ ${ }^{3}$ Especialista en Odontopediatría.Universidad Católica de Santa María, Arequipa-Perú. \\ *mavil_mc@hotmail.com
}

\begin{abstract}
Resumen
OBJETIVO: El propósito de este estudio fue comparar la influencia de la técnica de instrumentación rotatoria y convencional para pulpectomías en el tiempo operatorio y ansiedad de preescolares. MATERIAL Y MÉTODOS: el estudio fue cuasiexperimental, corte longitudinal, el tamaño de la muestra se distribuyó probabilísticamente en dos grupos con 20 sujetos de estudio (molares deciduos) en cada uno, quienes contaron con consentimiento y asentimiento informado, el análisis de los datos obtenidos se empleó el analista estadístico SPSS versión 22 y para la comprobación de la hipótesis se utilizó la prueba T de Student y Chi-cuadrado de McNemar. RESULTADOS: el tiempo operatorio en las pulpectomias de molares deciduas para la instrumentación rotatoria y convencional obtuvieron una mediana de $31,95(1,54)$ y 48,35 $(2,48)$ minutos respectivamente, la frecuencia cardiaca del niño durante la instrumentación rotatoria y convencional presentan una media de $70,65 \quad(3,36)$ y 85,30 (3,94) latidos por minuto (LPM) respectivamente, la saturación de oxígeno del niño durante la instrumentación rotatoria y convencional presentan una media de $91,55 \% \quad(0,89)$ y $91,70 \% \quad(1,26)$ respectivamente. CONCLUSIÓN: se encontró significancia estadística de la influencia en el tiempo operatorio y la ansiedad de preescolares con instrumentación rotatoria en pulpectomias de molares deciduas (IC del 95\%, $\mathrm{p}<=0,001$ ).
\end{abstract}

Palabras clave: Pulpectomía, Preescolares, Molares deciduos, Instrumentación rotatoria.

\begin{abstract}
AIM: The purpose of this study was to compare the influence of rotational and conventional instrumentation technique for pulpectomies on operative time and preschool anxiety. MATERIAL AND METHODS: The study was quasi-experimental, longitudinal cut, the sample size was probabilistically distributed in two groups with 20 study subjects (deciduous molars) in each one, for the analysis of the data obtained was used the statistical analyst SPSS version 22 and McNemar's Student's T-test and Chi-square test were used to test the hypothesis. RESULTS: The operative time in pulpectomies of deciduous molars for rotary and conventional instrumentation obtained a median of 31,95 (1,54) and 48,35 (2,48) minutes respectively, the child's heart rate during rotational and conventional instrumentation presented a mean of 70,65 (3,36) and 85,30 $(3,94)$ beats per minute (BPM) respectively, the oxygen saturation of the child during the rotational and conventional instrumentation presented a mean of $91,55 \%(0,89)$ and $91,70 \%(1,26)$ respectively. CONCLUSION: We found statistical significance of the influence on operative time and anxiety of preschoolers with rotational instrumentation in deciduous molar pulpectomies $(95 \% C I, p<=0,001)$.
\end{abstract}

Key words: Pulpectomy, Preschoolers, Molars deciduous, Rotary instrumentation.

\section{INTRODUCCIÓN}

Las enfermedades de la cavidad bucal ocupan el segundo lugar como motivo de consulta a nivel nacional con un 
$9,1 \%$, ocupando el primer lugar las infecciones agudas de las vías respiratorias que representan el $30 \%$ de las atenciones, caso que se repite en el departamento de Puno, asimismo se aprecia una alta severidad de la caries dental que refleja en el paciente pediátrico la necesidad terapéutica de pulpectomias en dentición decidua. ${ }^{1,2} \mathrm{Al}$ comparar las limas utilizadas en la instrumentación convencional con las de la instrumentación mecánica, estas últimas en los últimos años han evolucionado las técnicas mecánicas para la preparación del conducto radicular con el uso de instrumentos de níquel titanio, ganando popularidad el uso de limas rotatorias de níquel titanio por Endodoncistas y Cirujano Dentistas generales, por las características de mayor flexibilidad y condición de mantener la configuración original de conductos radiculares curvos con paredes delgadas características de los molares deciduos. ${ }^{3}$ La existencia de datos que evidencian que los usos de estos sistemas reducen las fallas relacionadas con la preparación de conductos radiculares permitiendo a la vez la conformación de una adecuada conicidad a gran velocidad ${ }^{4}$ y en dientes deciduos una adecuada conformación en forma de embudo del conducto permite realizar una obturación más uniforme. ${ }^{5}$ Un factor clínicamente relevante en la odontología pediátrica implica realizar procedimientos más rápidos manteniendo la calidad y seguridad de los tratamientos. ${ }^{6}$ Durante muchos años la ansiedad y el miedo en los niños al tratamiento dental ha sido una fuente de problemas para el manejo de paciente. ${ }^{7}$ El tratamiento endodóntico en los dientes primarios puede ser un reto y consumir mucho tiempo, especialmente durante la preparación del conducto, que es considerada uno de los pasos más importantes en la terapia del conducto radicular, las técnicas rotatorias mejoran el tiempo del tratamiento en los dientes primarios, ${ }^{5}$ por lo tanto, puede ser indicada en pacientes con problemas de manejo de conducta reduciendo la fatiga del paciente y del profesional. ${ }^{8}$ El dolor dental es la razón principal por el que los padres llevan al niño a consulta dental y usualmente por la severidad de la lesión la opción terapéutica es el tratamiento de conducto. Este trabajo de investigación evidenció cómo es que interactúan el tiempo operatorio, ansiedad fisiológica del niño (frecuencia cardiaca y saturación de oxígeno), manipulando la preparación del conducto con instrumentación rotatoria, ya que según sus características actuaria idóneamente en pulpectomias de molares deciduas.

\section{MATERIALES Y MÉTODOS}

El estudio fue experimental de corte longitudinal.El ámbito de estudio estuvo conformado por molares deciduas de preescolares del distrito de Paucarcolla, provincia de Puno Perú, departamento de Puno. El tamaño muestral se distribuyó probabilísticamente en dos grupos con 20 sujetos de estudio (molares deciduos) cada uno. Como criterios de inclusión se consideró niños con dos molares deciduas inferiores con diagnóstico de pulpitis irreversible, que radiográficamente tengan menos de $1 / 3$ de raíz reabsorbida y que requieran tratamiento de conducto. Este trabajo se realizó de acuerdo a normas bioéticas; ya que, se solicitó el consentimiento informado a la madre del niño y asentimiento del niño para el examen bucal, la pulpectomia con ambas técnicas de instrumentación, así como, para la publicación de los datos presentados en este trabajo. Para la recolección de datos, se empleó el método de observación para determinar el tiempo operatorio se usó un reloj minutero, mientras que, para la frecuencia cardiaca y la saturación de oxígeno se usó el pulsoxímetro, realizándose previamente una prueba piloto para medir la validez del contenido del instrumento con un $10 \%$ de la población par a la del estudio. El análisis y procesamiento de los datos se hizo mediante el analista estadístico SPSS versión 22 y Chi-cuadrado de McNemar con un intervalo de confianza del $95 \%(\mathrm{p}=0,05)$

\section{RESULTADOS}

Las pulpectomías en molares deciduas realizadas con instrumentación rotatoria y convencional tuvieron un tiempo operatorio promedio de $31,95(1,54)$ y $48,35(2,48)$ minutos respectivamente (Tabla 1). La frecuencia cardiaca del niño durante la instrumentación rotatoria y convencional presentan una media de $70.65(3,36)$ y $85.30(3,94)$ latidos por minuto respectivamente (Tabla 2). La saturación de oxígeno del niño $(\mathrm{SaO} 2)$ durante la instrumentación rotatoria y convencional presentan una media de $91,55 \%(0,89)$ y $91,70 \%(1,26)$ $\mathrm{SaO} 2$ cada una (Tabla 3).

\begin{tabular}{lcc}
\hline $\begin{array}{c}\text { ESTADÍSTICOS } \\
\text { DESCRIPTIVOS DE TIEMPO } \\
\text { OPERATORIO (MINUTOS) }\end{array}$ & $\begin{array}{c}\text { INSTRUMENTACIÓN } \\
\text { ROTATORIA } \\
\text { (GE) }\end{array}$ & $\begin{array}{c}\text { CONVENCIONAL } \\
\text { (GC) }\end{array}$ \\
\hline MEDIA & 31,95 & 48,35 \\
DESVIACIÓN TÍPICA & 1,54 & 2,48 \\
ERROR TÍPICO DE LA MEDIA & 0,344 & 0,554 \\
MÁXIMO & 35 & 53 \\
MÍNIMO & 30 & 45 \\
$\begin{array}{l}\text { MARGEN DE ERROR } \\
\text { PRUEBA T-STUDENT }\end{array}$ & $\mathrm{p}=<0,001$ & \\
\hline
\end{tabular}

Tabla 1. Tiempo operatorio instrumentación rotatoria y convencional.

\section{DISCUSIÓN}

La pulpectomía, se indica en dentición decidua que tengan lesiones severas de caries que comprometan tejido pulpar y posterior a la amputación de esta evidencien hiperemia aguda y/o crónica, necrosis pulpar con o sin afectación de la furca; que no evidencie reabsorción radicular patológica o fisiológica más de $1 \mathrm{~mm} .^{9}$ La pulpectomía necesita procedimientos eficaces que demanden un corto tiempo en el sillón 


\begin{tabular}{lcc}
\hline $\begin{array}{c}\text { ESTADÍSTICOS } \\
\text { DESCRIPTIVOS DE } \\
\text { FRECUENCIA CARDIACA } \\
\text { (LPM) }\end{array}$ & $\begin{array}{c}\text { INSTRUMENTACIÓN } \\
\text { ROTATORIA } \\
\text { (GE) }\end{array}$ & $\begin{array}{c}\text { CONVENCIONAL } \\
\text { (GC) }\end{array}$ \\
\hline MEDIA & 70,65 & $\mathbf{8 5 , 3 0}$ \\
DESVIACIÓN TÍPICA & 3,360 & 3,935 \\
ERROR TÍPICO DE LA MEDIA & 0,751 & 0,880 \\
MÁXIMO & 75 & 89 \\
MÍNIMO & 68 & 79 \\
MARGEN DE ERROR & & 0,000 \\
PRUEBA T-STUDENT & &
\end{tabular}

Tabla 2. Frecuencia cardíaca del paciente pediátrico durante la instrumentación rotatoria y convencional

\begin{tabular}{ccc}
\hline $\begin{array}{c}\text { ESTADÍSTICOS } \\
\text { DESCRIPTIVOS DE } \\
\text { SATURACIÓN DE OXÍGENO } \\
\text { (>87\%) }\end{array}$ & $\begin{array}{c}\text { INSTRUMENTACIÓN } \\
\text { ROTATORIA } \\
\text { (GE) }\end{array}$ & $\begin{array}{c}\text { CONVENCIONAL } \\
\text { (GC) }\end{array}$ \\
\hline MEDIA & $91,55 \%$ & $91,70 \%$ \\
DESVIACIÓN TÍPICA & $\mathbf{0 , 8 9}$ & 1,26 \\
ERROR TÍPICO DE LA MEDIA & 0,198 & 0,282 \\
MÁXIMO & $93 \%$ & $95 \%$ \\
MÍNIMO & $89 \%$ & $90 \%$ \\
MARGEN DE ERROR & & 0,67 \\
PRUEBA T-STUDENT & & \\
\hline
\end{tabular}

Tabla 3. Saturación de oxígeno durante la instrumentación rotatoria y convencional.

dental, la aprobación del paciente que certifique la funcionalidad de los dientes deciduos hasta el proceso de exfoliación natural. Para lo cual, se cuenta con procedimientos biomecánicos de preparación de conductos radiculares deciduos entre los que se tiene el uso de instrumentos rotatorios. Esta técnica se ha utilizado para realizar pulpectomías en dentición decidua, demostrando una disminución del tiempo operatorio y uniformidad en la obturación, generando un impacto positivo en la aceptación del niño hacia el tratamiento. ${ }^{5,8}$ Este estudio evidenció mejor tiempo operatorio utilizando instrumentos rotatoria para la preparación de conductos en molares deciduas que el uso de instrumentos convencionales, presentando por una media de $31,95(1,54)$ y $48,35(2,48)$ minutos respectivamente, IC del $95 \%$ y p $<0,001$. Estos resultados fueron similares a los encontrados por Porto A. y cols., ${ }^{10}$ que concluyen que el uso de instrumentos rotatorios para la conformación de los conductos radiculares disminuye significativamente el tiempo operatorio al compararlo con la instrumentación convencional, Crespo S. y Cols., ${ }^{5}$ refieren que el uso de instrumentación rotatoria en los dientes deciduos tiene varias ventajas en comparación con los instrumentos $\mathrm{K}$ manuales como la eficiencia en el tiempo de preparación y en la conformación del conducto radicular, para Katge F, y Cols, ${ }^{12}$ Kummer TR, y Cols, ${ }^{13}$ Musale PK, y Mujawar $\mathrm{SA}^{14}$ y Francinne MR, y Cols, ${ }^{15}$ la técnica rotatoria requirió menos tiempo, generó menos remoción de dentina y preparan más canales cónicos en los dientes deciduos, mejorando la cooperación del paciente, especialmente en niños pequeños. Para Azar y cols. ${ }^{16,17}$ existió significancia en la capacidad de limpieza de los conductos radiculares de las limas K-file comparadas a las limas usadas para instrumentación rotatoria y los tiempos menos extensos que se registraron con la técnica rotatoria. Así como, que el tiempo de trabajo fue más corto en dentición primaria o decidua que la dentición permanente. Para Arregui G y Cols. ${ }^{18}$ el uso de instrumentos rotatorios es un sistema adecuado y apto, que para facilitar el trabajo clínico debe introducirse gradualmente al momento de trabajar en dentición decidua, sin obviar alguno de sus inconvenientes.

El miedo leve y la ansiedad son esperables en el niño antes y durante la atención clínica, considerándolos con un desarrollo normal, pero se vuelven una preocupación y gran necesidad de tratamiento cuando estos son desproporcionados. La ansiedad dental es una expresión multisistémica a lo que se considera una amenaza o peligro, manifestándose como una experiencia individua y subjetiva que varía entre individuos. ${ }^{19}$ Para medir la ansiedad fisiológica que experimenta un individuo tenemos indicadores como frecuencia cardiaca y saturación de oxígeno, en el presente estudio al evaluar la frecuencia cardiaca de los niños se encontró efectividad significativa al contrastar la técnica de instrumentación rotatoria con la técnica de instrumentación convencional del conducto radicular (IC 95\%, p <0.00), sin embargo, al evaluar la saturación de oxigeno de los niños no se encontró diferencia estadística aceptable en la efectividad al contrastar la técnica de instrumentación rotatoria con la técnica de instrumentación convencional del conducto radicular. (IC $95 \%$ y un $\mathrm{p}<0,67)$; en este estudio se encontró una saturación de oxigeno de $91,55 \%(0,89)$; resultado que es corroborado por Gamponia M y Cols., ${ }^{20}$ que encontraron un promedio de $\mathrm{Sa} 02$ en la altura de $87,3 \%$ refiriendo que cuanto más pequeños eran los niños presentaban un promedio de $\mathrm{Sa} 02$ bajo, sugiriendo una adaptación fisiológica del individuo a través del tiempo a la altura, Mejía $\mathrm{H}$ y Col. ${ }^{21}$ hicieron una revisión sistemática de la literatura sobre hipoxemia en la altura y otra acerca de la epidemiología de la hipoxemia en niños con infección respiratoria; reportando como valores normales a $\mathrm{SaO} 2$ por encima de $85 \%$ en niños que residen a más de 2500 msnm. 


\section{CONCLUSIÓN}

El uso de instrumentos rotatorios en la preparación y conformación de los conductos radiculares para pulpectomias en molares deciduas permite una disminución del tiempo de trabajo, favorece la participación favorable del niño con una disminución de los niveles de ansiedad y el posible cansancio del niño, reduciendo indirectamente la fatiga del profesional y aumentando de esta forma el éxito clínico.

\section{Referencias}

1 Red de Salud Puno. Morbilidad general por grupos de enfermedad: Red Puno; 2015 [citado 13 Abril 2015].

2 Ministerio de Salud. Plan Nacional Bicentenario el Perú hacia el 2021, aprobado por el Acuerdo Nacional. 2011.

3 Kuo, C., Wang, Y., Chang, H., Huang, G., Lin, C., Li, U. Application of Ni-Ti rotary files for pulpectomy in primary molars. J Dent SciVolume 1, Pages 10-15

4 Ochoa T, Mendez V, Martínez A y Pozos A. Instrumentación rotatoria en dientes temporales. Reporte de un caso. Odontol Pediatr. 2011;10:(1)51-54.

5 Crespo S., Cortes O., Garcia C., Perez L. Comparison between rotary and manual instrumentation in primary teeth. J. Clin. Pediatr. Dent. 2008;32:295-298.

6 Sageena George, S. Anandaraj, Jyoti S. Issac, Sheen A. John, Anoop Harris. Rotary endodontics in primary teeth - A review. The Saudi Dental Journal (2016) 28,12-17.

7 Sarnat H. Arad P. Hanauer D. Shohami E.Communication strategies used during pediatric dental treatment: a pilot study. American Academy of Pediatric Dentistry. 2001:23:3.

8 Hülsmann M, Herbst U, Schäfers F. Comparative study of root-canal preparation using Lightspeed and Quantec SC rotary NiTi instruments. International Endodontic Journal .Volume 36, Issue 11 November 2003 Pages 748-756

9 Villena H. Terapia Pulpar. Primera edición: Lima Perú; Universidad Peruana Cayetano Heredia. 2001.

10 Porto A, Ramos A, Simionatto C, Rivas JC, Filho I. Evaluación de la instrumentación de conductos radiculares con limas manuales y rotatorias de níquel-titanio aspecto morfológico de las limas antes y después. Acta Odontológica Venezolana. 2008; 46(3):278-283.

11 Crespo S., Cortes O., Garcia C., Perez L. Comparison between rotary and manual instrumentation in primary teeth. $\mathrm{J}$. Clin. Pediatr. Dent. 2008;32:295-298. [PubMed]

12 Katge F., Patil D., Poojari M., Pimpale J., Shitoot A., Rusawat B. Comparison of instrumentation time and cleaning efficacy of manual instrumentation, rotary systems and reciprocating systems in primary teeth: an vitro study. J. Indian Soc. Pedod. Prev. Dent. 2014;32:311-316. [PubMed]

13 Kummer T.R., Calvo M.C., Cordeiro M.M., de Sousa Vieira R., de Carvalho Rocha M.J. Ex vivo study of manual and rotary instrumentation techniques in human primary teeth. Oral Surg. Oral Med. Oral Pathol. Oral Radiol. Endod. 2008;105:84-92. [PubMed]
14 Musale P.K., Mujawar S.A. Evaluation of the efficacy of rotary versus hand files in root canal preparation of primary teeth in vitro using CBCT. Eur. Arch. Paediatr. Dent. 2014;15:113-120. [PubMed]

15 Francinne M. R., Modesto A., Faraco I.M. Manual and rotary instrumentation techniquesfor root canal preparation. DENTISTRY 3000. 2014;2(1)

16 Azar MR, Mokhtare M. Rotary Mtwo system versus manual K-file instruments: Efficacy in preparing primary and permanent molar root canals. Indian J Dent Res 2011;22:363

17 Azar MR, Safi L, Nikaein A. Comparison of the cleaning capacity of Mtwo and Pro Taper rotary systems and manual instruments in primary teeth. Dental Research Journal. 2012;9(2):146-151.

18 Arregui M, Guinot F, Sáez S, Bellet LJ. Instrumentación mecánica en dentición temporal. ODONTOL PEDIÁTR. Madrid. 2005;13(3):102-107.

19 Klingberg G y Broberg A. Dental fear/anxiety and dental behavior management problems in children and adolescents: a review of prevalence and concomitant psychological factors. Int J Paediatr Dent. 2007;17(6):391-406.

20 Gamponia M, Yugar F, Babaali H, Gilman R. Valores de referencia para pulsoximetría en la altura. Archivos del Hospital La Paz. 2004. Mayo, 1(1)

21 Mejía H y Mejía M. Oximetría de pulso. Rev. bol. ped. [Internet]. 2012 [citado 2016 Mar 05];51(2):149-155.

Recibido: 23 de Junio de 2018.

Aceptado: 23 de Julio de 2018. 